\section{RÉFÉRENCES}

1. Grennberg S, Grinstein S. Phagocytosis and innate immunity. Curr Opin Immunol 2002 ; 14 : 136-45.

2. Sahu A, Lambris JD. Structure and biology of complement protein C3, a connecting link between innate and acquired immunity. Immunol Rev $2001 ; 180: 35-48$.

3. Booth JW, Trimble WS, Grinstein S. Membrane dynamics in phagocytosis. Semin Immunol 2001 ; 13 : 357-64.
4. Babior BM, Lambeth JD, Nauseef $W$. The neutrophil NADPH oxidase. Arch Biochem Biophys 2002 ; 397 : 342-4.

5. Babior BM, Kipnes RS, Curnutte JT. Biological defence mechanisms: the production by leukocytes of superoxyde, a potential bactericidal agent. J Clin Invest 1973 ; 52 : 741-4.

6. Hampton MB, Kettle AJ, Winterbourn CC. Inside the neutrophil phagosome: oxidants, myeloperoxydase and bacterial killing. Blood 1998 ; 92 : 3007-17.
7. Thrasher AJ, Keep NH, Wientjes F, Segal, AW. Chronic granulomatous disease. Biochem Biophys Acta 1994 ; 1227 : 1-24.

8. Aratani $y$, Koyama H, Nyui S, Suzuki K, Kura F, Maeda N. Severe impairment in early host defense against Candida albicans in mice deficient in myeloperoxydase. Infect Immun 1999; 67 : 1828-36.

9. Reeves EP, Lu H, LortatJacob $\mathrm{H}$, et al. Killing activity of neutrophils is mediated through activation of proteases by $\mathrm{K}^{+}$flux. Nature 2002 ; 416 : 291-7.

10. Hardingham TE, Fosang AJ. Proteoglycans: many forms and many functions. FASEB J $1992 ; 6$ : 861-70.

11. Esko JD, Lindahl U. Molecular diversity of heparan sulfate. J Clin Invest $2001 ; 108$ : 169-73.

12. Lortat-Jacob H, Grosdidier A, Imberty A. Structural diversity of heparan sulfate binding domains in chemokines.

Proc Natl Acad Sci USA 2002 ; 99 : 1229-34.

\title{
NOUVELL $\varepsilon$
}

\section{Des moustiques capables d'interrompre le cycle du Plasmodium}

transforme en un ookinète doué de motilité. Ce dernier interagit avec la face luminale de l'épithélium stomacal qu'il traverse, et se transforme sur la surface externe de l'estomac en oocyste. Au sein de l'oocyste sont produits de nombreux sporozoïtes, qui sont libérés au bout de quelques jours dans l'hémocœle du moustique. Ces sporozoïtes entrent en contact avec l'épithélium
Département de génétique, développement et pathologie moléculaires, Inserm U.567, 24, rue du Faubourg SaintJacques, 75014 Paris, France. labie@cochin.inserm.fr paludisme reste une des grandes causes de morbidité et de mortalité dans le monde: 300 à 500 millions de nouveaux cas par an, près d'un milliard d'êtres humains infectés, 1 à 3 millions de morts annuelles, principalement chez les jeunes enfants, en zone tropicale. Le cycle de vie du parasite responsable de cette maladie, Plasmodium, est complexe et alterne entre l'hôte humain et le moustique vecteur. Cette maladie affecte principalement les populations défavorisées. L'extrême variabilité génétique du Plasmodium complique la mise en route d'une stratégie vaccinale et, par ailleurs, les moustiques ont développé des résistances aux insecticides avec lesquels on avait cru pouvoir les éradiquer. II est donc probable qu'on ne trouvera pas une recette miracle unique, et qu'il faudra combiner diverses approches pour contrôler cette maladie, qu'il s'agisse d'approches thérapeutiques et/ou prophylactiques [1]. De multiples approches pro- phylactiques ont été envisagées qui cherchent à interrompre le cycle de Plasmodium falciparum.

On a le plus souvent ciblé les différentes étapes du cycle de développement du parasite chez l'homme. Une autre stratégie est présentée dans Nature par une équipe incluant des chercheurs de la Case Reserve University (Ohio, USA) et de l'Université de Bayreuth (Allemagne). Les auteurs montrent pour la première fois qu'il est envisageable d'interrompre la transmission du parasite au cours de son développement chez des moustiques transgéniques [2] (Figure 1), les étapes du cycle du Plasmodium. Une récente revue de la même équipe fait le point sur les phases se développant chez le moustique [3] (Figure 1). Au cours de la piqûre d'un individu infecté, le moustique prélève des gamétocytes mâles et femelles, précurseurs des gamètes, qui fusionnent dans l'estomac de l'insecte pour former un zygote qui se des glandes salivaires, qu'ils envahissent et peuvent alors être transmis à un nouvel hôte humain à l'occasion d'une piqûre. Pour interrompre cette phase du cycle, il fallait savoir à laquelle des protéines de l'épithélium de l'estomac et/ou des glandes salivaires se fixe spécifiquement le parasite avant de les traverser. Récemment, le criblage d'une banque d'expression obtenue à partir d'un phage a permis aux chercheurs de la Case Western University de caractériser un peptide de 12 acides aminés, appelé SMI (salivary gland-and midgut binding peptide 1) qui se fixe spécifiquement aux épithéliums de l'estomac et des glandes salivaires du moustique [4]. Ingéré par le moustique, le peptide SMI inhibe fortement l'invasion de l'estomac par les ookinètes ; injecté dans l'hémocœle de l'insecte, ce même peptide 
inhibe également l'invasion des glandes salivaires par les sporozoïtes. Ce résultat suggère que le peptide $S M I$ bloque les récepteurs reconnus par le parasite au niveau de l'estomac et des glandes salivaires.

Le but des auteurs du travail actuel a été d'obtenir un moustique transgénique exprimant le peptide SMl. Ils ne sont pas les premiers à avoir envisagé la production de moustiques transgéniques. Les chercheurs de I'University of California (Irvine, CA, USA) avaient fait exprimer au moustique Aedes aegypti des anticorps contre le parasite, mais la technologie utilisée ne permettait qu'une expression transitoire de ces anticorps chez le moustique sans production de lignées transgéniques stables [5]. Dans l'étude actuelle, les auteurs sont partis de la caractérisation préalable du gène de la carboxypeptidase du moustique [6]. Le promoteur du gène est, en effet, activé par le repas de sang que fait le moustique et la carboxypeptidase est exprimée dans la lumière stomacale dans les heures qui suivent, en même temps que se forme l'ookinète. La stratégie employée pour inhiber le développement du Plasmodium a été la construction d'un gène de synthèse $\left(A g C P[S M I]_{4}\right)$ se

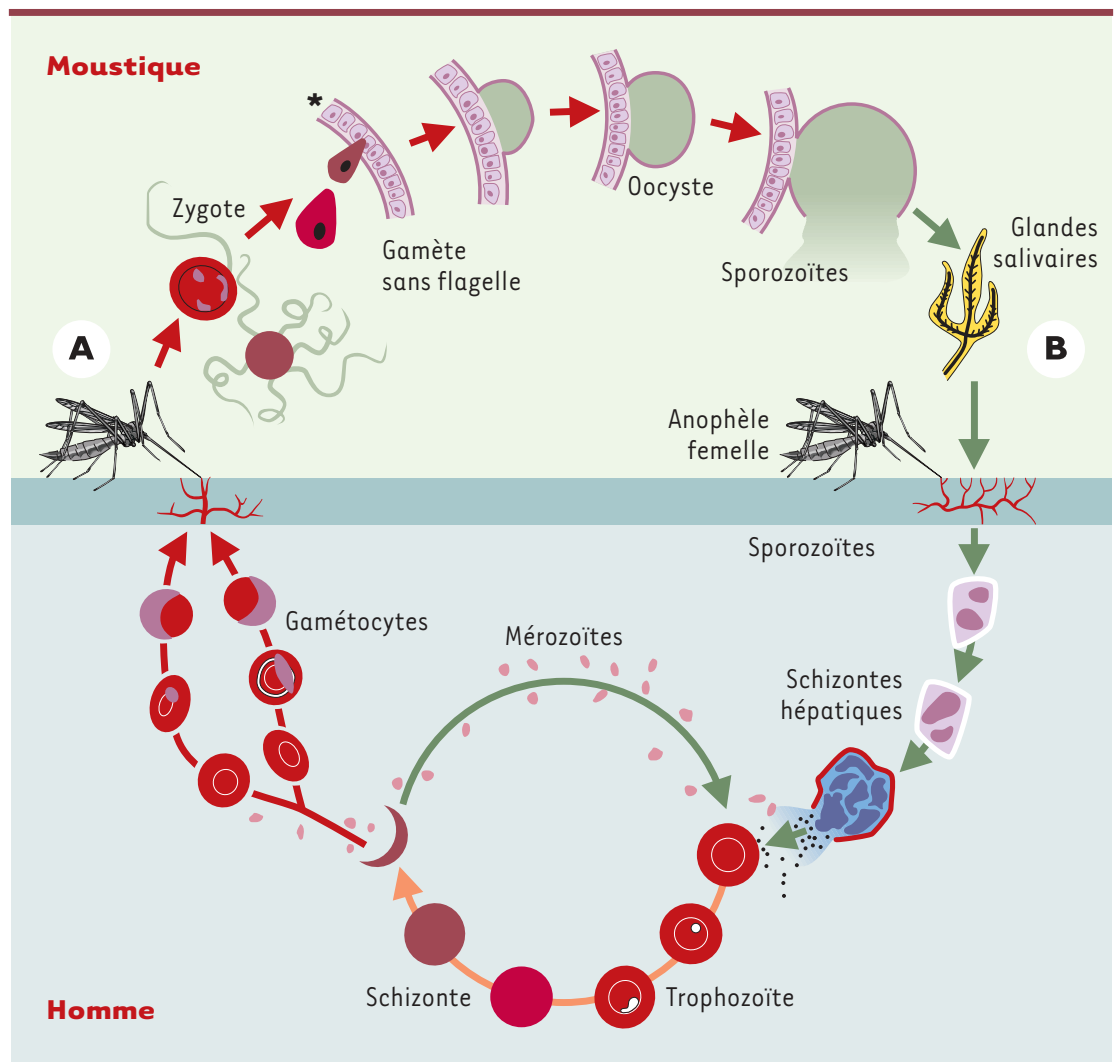

Figure 1. Étapes successives du cycle du Plasmodium. En bas, les étapes qui ont lieu dans l'organisme humain, en haut celles qui ont lieu chez le moustique et qui font l'objet de cet article. $\varepsilon$ n $A$, la première piqûre : l'insecte prélève les gamétocytes, qui deviennent les gamètes, le même repas déclenchant la production de SMI dans la cavité stomacale chez le moustique transgénique $(*)$. La fusion des gamètes donne lieu au zygote, qui devient un ookinète. C'est le passage de cet ookinète à travers l'épithélium stomacal vers l'hémocœle, et sa transformation en oocyste, qui sont inhibés par SMI chez le moustique transgénique. Après libération des sporozoïtes de l'oocyste, il y a normalement passage de ces sporozoïtes dans la glande salivaire de l'insecte, rendant infectieuse sa piqûre (en B). On peut imaginer pour ce passage vers les glandes salivaires un processus d'inhibition semblable à celui qui a été montré concernant le passage de l'épithélium stomacal.

liant au promoteur codant pour la protéine carboxypeptidase et sa séquence signal à 4 unités liées de SMl. Après introduction dans un vecteur PiggyBac (transposon capable de s'insérer dans le génome du moustique), cette construction a été utilisée pour obtenir des lignées transgéniques stables d'Anopheles stephensi. Plusieurs familles de moustiques issues de ces lignées et présentant apparemment une vitalité normale ont été étudiées. On a constaté par Southern blot que le transgène était intégré chaque fois à une position différente, et l'analyse de I'ARN a montré une forte expression du gène après chaque ingestion de sang. La protéine recombinante, détectée grâce à un anticorps fluorescent, était liée à la face luminale de l'épithélium stomacal 6 h à 24 h après le repas sanguin, sa présence s'atténuant fortement après $36 \mathrm{~h}$. Les moustiques transgéniques et des moustiques témoins ont été nourris sur les mêmes souris infectées par Plasmodium. On a observé chez les premiers une diminution $d u$ nombre d'oocystes par moustique, dépassant en moyenne $80 \%$ (68,7 à 94,9\%), mais aussi une réduction du nombre de moustiques porteurs d'oocystes. Le nombre de sporozoïtes observé au bout de 25 jours chez les moustiques transgéniques était beaucoup plus faible que chez les témoins ( 1,7 à $19 \%$ seulement), diminuant fortement leur compétence à transmette le parasite à d'autres souris. Cette dernière constatation serait évidemment d'une importance particulière sur le terrain. Les auteurs notent enfin que toutes ces expériences ont été faites avec des moustiques hétérozygotes pour le transgène, et que des résultats encore meilleurs seraient à attendre de moustiques homozygotes.

L'ensemble de ces résultats représente incontestablement une étape majeure dans la lutte multiforme qui est menée contre le paludisme. On ne saurait cependant ignorer tous les défis et difficultés. Ce qui est démontré dans le modèle souris sera-t-il vrai chez l'homme? Des observations faites en laboratoire seront-elles vérifiées sur le terrain? Qu'en sera-t-il de 
la diversité génétique et de la mutabilité connue du Plasmodium? Cette approche, comme d'autres, sélectionnera-t-elle des variants du parasite capables d'utiliser d'autres voies de développement chez le moustique ? II est sûrement nécessaire, dans cette optique, de pouvoir introduire chez le moustique des modifications génétiques jouant sur plusieurs mécanismes simultanés. Comment, aussi, diffuser sur le terrain une population de moustiques porteurs de gènes étrangers ? On est sans doute encore loin de l'application. Les avancées génomiques actuelles devraient fournir aux chercheurs dans un futur proche toutes les séquences de l'homme, du parasite et du vecteur. Des approches multiples et coordonnées font espérer que l'on maîtrisera un jour ce terrible fléau qu'est le paludisme.

Le travail tout récent de chercheurs japonais de l'École de Médecine de la Mie University, Edobashi, Tsu, sans relation avec le précédent, mérite d'être signalé, car il relève de la même préoccupation : les étapes du développement du parasite chez le moustique vecteur [7]. MAEBL a été décrite comme membre d'une famille très conservée de protéines de Plasmodium, impliquées dans les propriétés adhésives des mérozoïtes et dans l'invasion du globule rouge [8]. Le nom $M A E B L$ est en rapport avec une structure dont certains éléments sont comparables à la protéine DBL (Duffy binding protein) et d'autres à l'antigène de membrane AMA-1 (apical membrane antigen 1). Étudiant $M A \varepsilon B L$, l'équipe japonaise a observé une production spécifique de cette protéine par les sporozoïtes contenus dans les oocystes. La protéine est localisée dans les micronèmes et vraisemblablement sécrétée. Les sporozoïtes isolés de glandes salivaires en sont dépourvus. L'inactivation du gène correspondant $a$ montré que MAEBL est essentielle pour l'infection des glandes salivaires. Ce pourrait être une molécule parasitaire reconnaissant spécifiquement un récepteur des glandes salivaires permettant la traversée de l'épithélium par les sporozoïtes. Cette étape-là, aussi, pourra-telle être utilisée pour obtenir des moustiques incapables de transmettre l'agent du paludisme? $\diamond$

Mutant mosquitos to fight Plasmodium

\section{REMERCIEMENTS}

Nous remercions $R$. Brosch (Institut Pasteur, Paris, France) pour sa relecture du texte.

\section{NOUVELLE}

Recherche ligand
désespérément...
Patricia Ducy

$>$ On sait depuis maintenant huit ans que trois des quatre récepteurs des facteurs de croissance fibroblastiques (FGFR) jouent un rôle essentiel dans le contrôle de la squelettogenèse, quoique réglant des processus différents $[1,2]$. En effet, l'existence de mutations activatrices dans les gènes codant pour le FGFRl et le
FGFR2 entraîne une fusion prématurée des sutures crâniennes (craniosynostose), indiquant donc que les FGF correspondants sont impliqués dans le contrôle de l'ostéogenèse [3]. En revanche, les mutations activant de façon constitutive le FGFR3 entraînent des anomalies de croissance des os longs, inhibant avec plus ou al. Virus-expressed, recombinant single-chain antibody blocks sporozoite infection of salivary glands in Plasmodium gallinaceum-infected Aedes aegypti. Am J Trop Med Hyg 2000 ; 62 : 427-33.

6. Edwards MJ, Moskalyk LA, Donelly-Doman $M$, et al. Characterization of a carboxypeptidase A gene from the mosquito, Aedes aegypti. Insect Mol Biol $2000 ; 9: 33-8$.

7. Kariu T, Yuda M, Yano K, Chinzei Y. MAEBL is essential for malarial sporozoïte infection of the mosquito salivary gland. J Exp Med 2002 ; 195 :

1317-23.

8. Kappe SHI, Noe AR, Fraser TS, Blair PL, Adams JH. A family of chimeric erythrocyte binding proteins of malaria parasites. Proc Natl Acad Sci USA 1998 ; 95 : 1230-5.

\author{
Department of Molecular \\ and Human Genetics \\ and Department of Medicine, \\ Baylor College of Medicine, \\ One Baylor Plaza, Houston, \\ TX 77030, États-Unis.
}

moins de sévérité la différenciation des chondrocytes formant les cartilages de croissance. La production et l'analyse de multiples modèles murins, qu'il s'agisse de souris transgéniques surexprimant les versions sauvages ou mutantes de ces récepteurs, ou encore de souris dépourvues de ces récepteurs, a, depuis, précisé 